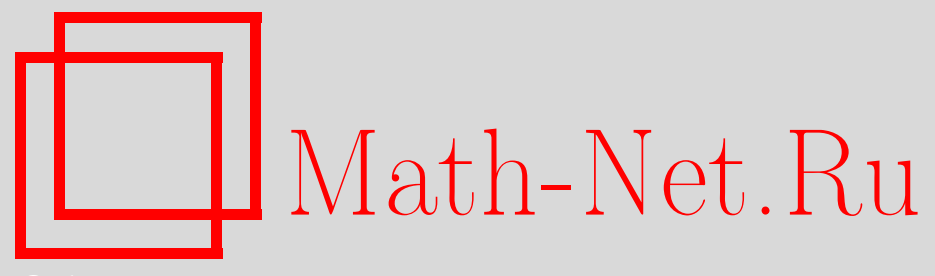

А. В. Еремин, Е. В. Стефанюк, А. Ю. Рассыпнов, А. Э. Кузнецова, Нестационарный теплообмен в цилиндрическом канале при ламинарном течении жидкости, Вестн. Сам. гос. техн. ун-та. Сер. Физ.-мат. науки, 2013, выпуск 4(), 122-130

DOI: https://doi.org/10.14498/vsgtu1211

Использование Общероссийского математического портала MathNet.Ru подразумевает, что вы прочитали и согласны с пользовательским соглашением

http://www.mathnet.ru/rus/agreement

Параметры загрузки:

IP : 54.162 .27 .143

26 апреля 2023 г., $11: 25: 53$

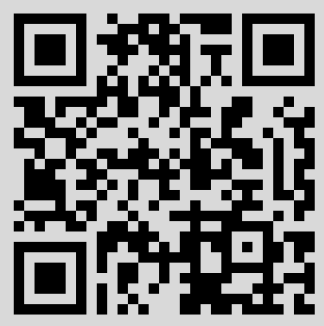


Вестн. Сам. гос. техн. Ун-та. Сер. Физ.-мат. науки. 2013. № 4 (33). С. $122-130$

УДК 517.958:536.24

\title{
НЕСТАЦИОНАРНЫЙ ТЕПЛООБМЕН В ЦИЛИНДРИЧЕСКОМ КАНАЛЕ ПРИ ЛАМИНАРНОМ ТЕЧЕНИИ ЖИДКОСТИ
}

\author{
А. В. Еремин, Е. В. Стефанюк, А. Ю. Рассыпнов, А. Э. Кузнецова
}

Самарский государственный технический университет,

Россия, 443100, Самара, ул. Молодогвардейская, 244.

E-mails: a.v.eremin@list.ru, stef-kate@yandex.ru, kuznetsovaae@rambler.ru

С использованием двукратного интегрального преобразования Лапласа-Карсона и ортогонального метода Бубнова-Галёркина получено аналитическое решение нестационарной задачи теплообмена при течении жидкости в иилиндрическом канале. Показано, что решение задачи разделяется на две составляюшие - стаиионарную и нестационарную, каждая из которых применима лишь в определённом диапазоне временной и пространственной координат. Для стационарной задачи (задача Гретиа-Нуссельта) путём совместного применения методов Фуръе и Бубнова-Галёркина с использованием дополнительных граничных условий получено приближённое аналитическое решение, позволяющее выполнять оценку температурного состояния жидкости при малых значениях пространственной переменной, направленной вдоль течения потока. Получение таких результатов на основе исполъзовании известных точных аналитических методов ввиду плохой сходимости бесконечных рядов получаемых решений не представляется возможным.

Ключевые слова: иилиндрический канал, задача Гретиа-Нуссельта, интегральное преобразование Лапласа-Карсона, метод Фуръе, дополнительные условия, задача Штурма-Лиувилля, аналитическое решение.

В процессе решения краевой задачи нестационарной теплопроводности при ламинарном течении жидкости в цилиндрическом канале происходит её физически обоснованное разделение на две задачи - нестационарную и стационарную, границы применимости которых определяются соотношениями между временной и продольной пространственной переменными. Дальнейшее решение каждой из полученных задач, определённых в строго фиксированных границах указанных переменных, выполняется раздельно. Нестационарная часть задачи представляет собой задачу теплопроводности для сплошного цилиндра (теплообмен протекает как бы в неподвижной жидкости), точные аналитические решения которой известны. Наибольшую трудность в данной случае представляет решение стационарной задачи с учётом движения жидкости (задача Гретца-Нуссельта). Эта задача впервые была решена Гретцем в 1885 г. [1]. Независимо от Гретца в 1910 г. её также решил Нуссельт [2]. Уточнённое решение задачи Гретца-Нуссельта дано в [3]. Отметим, что приведённое в [3] решение представляет собой бесконечный функциональный ряд, плохо сходящийся при малых значениях продольной координаты $(x<0,01)$, то есть в области, где исследования изменения температуры представляют наибольший интерес. Отметим также, что в зависимости от

Антон Владимирович Еремин, аспирант, каф. теоретических основ теплотехники и гидродинамики. Екатерина Василъевна Стефанюк (д.т.н.), доцент, каф. теоретических основ теплотехники и гидромеханики. Александр Юръевич Рассыпнов, аспирант, каф. теоретических основ теплотехники и гидромеханики. Анастасия Эдуардовна Кузнецова, аспирант, каф. теоретических основ теплотехники и гидромеханики. 
величины поперечной координаты решение содержит функции Бесселя различного (в том числе и дробного) порядка. Такое решение малопригодно для инженерных приложений. В связи с этим разработка приближённых аналитических методов решения подобных задач имеет как научную ценность, так и практическое значение. Одно из таких решений путём совместного использования интегральных преобразований Лапласа и ортогонального метода Бубнова-Галёркина получено П. В. Цоем [4]. Решение найдено лишь в третьем приближении. Получение решения в последующих приближениях ограничивается проблемой, связанной с трудностями решения систем алгебраических линейных уравнений в общем виде, а также перехода от изображения искомой функции к её оригиналу.

В настоящей работе излагается метод получения решения задачи ГретцаНуссельта, основанный на совместном использовании метода Фурье и ортогонального метода Бубнова-Галёркина. Его отличительными особенностями являются простота получаемых аналитических выражений и возможность нахождения решения для малых значений продольной пространственной переменной [5-7].

Рассмотрим полную последовательность получения аналитического решения нестационарной задачи теплообмена при течении жидкости в цилиндрическом канале. Введём следующие допущения: течение жидкости и процесс теплообмена стационарны; жидкость несжимаема, её физические свойства постоянны; профиль скорости не изменяется по длине трубы; температура жидкости на входе в трубу неизменна по сечению и равна $t_{0}$; температура внутренней поверхности стенки трубы постоянна и равна $t_{\mathrm{c}}$, причём

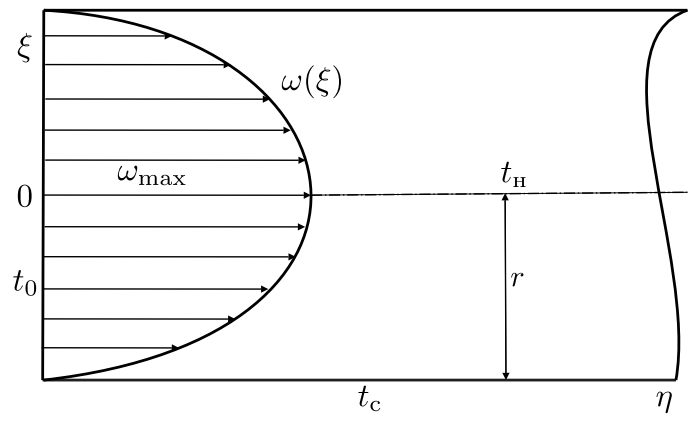

Рис. 1. Схема стабилизированного ламинарного течения жидкости в круглой трубе $t_{\mathrm{c}} \neq t_{0}$; начальная температура жидкости равна $t_{\mathrm{H}}$; внутренние источники тепла и диссипацию энергии не учитываем; переносом теплоты за счёт теплопроводности вдоль оси трубы пренебрегаем (рис. 1).

Математическая постановка задачи имеет следующий вид $[3,4]$ :

$$
\begin{gathered}
\frac{\partial t(\xi, \eta, \tau)}{\partial \tau}+\omega_{x} \frac{\partial t(\xi, \eta, \tau)}{\partial \eta}=\frac{a}{\xi} \frac{\partial t(\xi, \eta, \tau)}{\partial \xi}+a \frac{\partial^{2} t(\xi, \eta, \tau)}{\partial \xi^{2}}, \\
\tau>0, \quad \eta>0, \quad 0<\xi<r ; \\
t(\xi, \eta, 0)=t_{\mathrm{H}} ; \quad t(\xi, 0, \tau)=t_{0} ; \quad t(r, \eta, \tau)=t_{\mathrm{c}} ; \quad \frac{\partial t(0, \eta, \tau)}{\partial \xi}=0 .
\end{gathered}
$$

Здесь $t$ - температура; $\eta, \xi$ - продольная и поперечная координаты; $r$ - радиус трубы; $t_{0}$ - температура жидкости на входе в трубу (при $\left.\eta=0\right) ; t_{\mathrm{c}}$ температура стенки; $t_{\mathrm{H}}$ - начальная температура; $a$ - коэффициент температуропроводности; $\omega_{x}=2 \omega_{\mathrm{cp}}\left(1-\xi^{2} / r^{2}\right)$ - распределение скорости по координате $\xi ; \omega_{\mathrm{cp}}=\omega_{\max } / 2-$ средняя скорость; $\omega_{\max }-$ максимальная скорость ламинарного течения (рис. 1). 
Введём следующие безразмерные переменные и параметры:

$$
\Theta=\frac{t-t_{\mathrm{c}}}{t_{0}-t_{\mathrm{c}}} ; \quad y=\frac{\xi}{r} ; \quad x=\frac{1}{2} \frac{\eta}{\mathrm{Pe} r} ; \quad \mathrm{Pe}=\frac{\omega_{\mathrm{cp}} r}{a} ; \quad \text { Fo }=\frac{a \tau}{r^{2}} .
$$

Здесь $\Theta$ - относительная избыточная температура; $y$ - безразмерная поперечная координата; $x$ - безразмерная продольная координата; Ре - число Пекле; Fo - число Фурье.

С учётом принятых обозначений задача (1), (2) записывается так:

$$
\begin{gathered}
\frac{\partial \Theta(y, x, \mathrm{Fo})}{\partial \mathrm{Fo}_{0}}+\left(1-y^{2}\right) \frac{\partial \Theta(y, x, \mathrm{Fo})}{\partial x}=\frac{1}{y} \frac{\partial \Theta(y, \eta, \mathrm{Fo})}{\partial y}+\frac{\partial^{2} \Theta(y, \eta, \mathrm{Fo})}{\partial y^{2}} \\
\text { Fo }>0, \quad x>0, \quad 0<y<1 ; \\
\Theta(y, x, 0)=T_{\mathrm{H}} ; \quad \Theta(y, 0, \mathrm{Fo})=T_{0}=1 ; \quad \Theta(1, x, \mathrm{Fo})=0 ; \quad \frac{\partial \Theta(0, x, \mathrm{Fo})}{\partial y}=0,
\end{gathered}
$$

где $T_{\mathrm{H}}=\left(t_{\mathrm{H}}-t_{\mathrm{c}}\right) /\left(t_{0}-t_{\mathrm{c}}\right)$.

Для решения задачи (3), (4) применим двукратное преобразование Лапласа-Карсона по безразмерному времени Fо и относительной координате $x$ [4]:

$$
\begin{gathered}
T^{*}(y, S, \text { Fo })=S \int_{0}^{\infty} \Theta(y, x, \text { Fo }) \exp (-S x) d x \\
T(y, S, P)=P \int_{0}^{\infty} T^{*}(y, S, \text { Fo }) \exp (-P \text { Fo }) d \text { Fo, }
\end{gathered}
$$

где $P, S$ - параметры двукратного преобразования Лапласа-Карсона.

Соотношение (6) с учётом (5) примет вид

$$
T(y, S, P)=P S \int_{0}^{\infty} \int_{0}^{\infty} \Theta(y, x, \mathrm{Fo}) \exp (-S x-P \mathrm{Fo}) d x d \mathrm{Fo} .
$$

Задача (3), (4) в области изображений запишется следующим образом:

$$
\begin{gathered}
P\left[T(y, S, P)-T_{\mathrm{H}}\right]+S\left(1-y^{2}\right)\left[T(y, S, P)-T_{0}\right]- \\
-\frac{1}{y} \frac{\partial T(y, S, P)}{\partial y}-\frac{\partial^{2} T(y, S, P)}{\partial y^{2}}=0 ; \\
T(1, S, P)=0 ; \quad \frac{\partial T(0, S, P)}{\partial y}=0 .
\end{gathered}
$$

Решение задачи (7), (8) в соответствии с ортогональным методом Бубнова-Галёркина в первом приближении принимается в виде

$$
T(y, S, P)=\nu_{1}(S, P) \varphi_{1}(y)
$$

где $\nu_{1}(S, P)$ - неизвестный коэффициент-изображение; $\varphi_{1}(y)-$ координатная функция, определяемая соотношением

$$
\varphi_{1}(y)=1-y^{2}
$$


Очевидно, что соотношение (9) при использовании координатной функции вида (10) удовлетворяет граничным условиям (8). Для определения неизвестного коэффициента $\nu_{1}(S, P)$ составим невязку уравнения $(7)$ и потребуем ортогональности невязки и координатной функции $\varphi_{1}(y)$ :

$$
\begin{aligned}
\int_{0}^{1}\left\{y P\left[T(y, S, P)-T_{\mathrm{H}}\right]+y S\right. & \left(1-y^{2}\right)\left[T(y, S, P)-T_{0}\right]- \\
& \left.-\frac{\partial T(y, S, P)}{\partial y}-y \frac{\partial^{2} T(y, S, P)}{\partial y^{2}}\right\} \varphi_{1}(y) d y=0 .
\end{aligned}
$$

Подставляя (10) в (9), преобразуем полученное выражение:

$$
\begin{aligned}
\int_{0}^{1}\left\{y P\left[\nu_{1}(S, P)\left(1-y^{2}\right)-T_{\mathrm{H}}\right]\right. & +y S\left(1-y^{2}\right)\left[\nu_{1}(S, P)-T_{0}\right]+ \\
& \left.+2 \nu_{1}(S, P) y+2 \nu_{1}(S, P) y\right\}\left(1-y^{2}\right) d y=0 .
\end{aligned}
$$

Вычисляя интегралы в (11), относительно неизвестного коэффициента $\nu_{1}(S, P)$ получаем

$$
\nu_{1}(S, P)=\frac{P F_{1}+S F_{2}}{P F_{3}+S F_{4}+F_{5}},
$$

где

$$
\begin{gathered}
F_{1}=T_{\mathrm{H}} \int_{0}^{1} y\left(1-y^{2}\right) d y, \quad F_{2}=T_{0} \int_{0}^{1} y\left(1-y^{2}\right)^{2} d y, \quad F_{3}=\int_{0}^{1} y\left(1-y^{2}\right)^{2} d y, \\
F_{4}=\int_{0}^{1} y\left(1-y^{2}\right)^{3} d y, \quad F_{5}=4 \int_{0}^{1} y\left(1-y^{2}\right) d y .
\end{gathered}
$$

В пространстве оригиналов соотношение (9) запишется в виде

$$
\Theta(y, x, \text { Fo })= \begin{cases}\frac{F_{1}}{F_{3}} \exp \left(-\frac{F_{5}}{F_{3}} \text { Fo }\right) \varphi_{1}(y) & \text { при } x>\frac{F_{4}}{F_{3}} \text { Fo }, \\ \frac{F_{2}}{F_{3}} \exp \left(-\frac{F_{5}}{F_{4}} x\right) \varphi_{1}(y) & \text { при } x<\frac{F_{4}}{F_{3}} \text { Fo. }\end{cases}
$$

Верхняя строка формулы (12) совпадает с решением в первом приближении задачи (3), (4) при равенстве нулю конвективного члена (второе слагаемое в левой части) уравнения (3), то есть с решением нестационарной задачи теплопроводности для бесконечного цилиндра.

Нижняя строка формулы (12) совпадает с решением в первом приближении стационарной задачи конвективного теплообмена, то есть при равенстве нулю первого члена в левой части уравнения (3).

Таким образом, для областей теплообменника, которых не достигло воз-

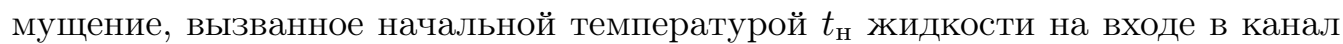
$($ при $x=0)$, нестационарный теплообмен происходит как бы в неподвижной жидкости, то есть перенос теплоты происходит только за счёт теплопроводности. Данная задача представляет собой нестационарную задачу теплопроводности для бесконечного цилиндра, классические точные аналитические решения которой известны. 
Для областей теплообменника, подверженных влиянию температурных условий на входе в трубу (жидкость при течении достигла этих областей), теплообмен не зависит от начальной температуры $t_{0}$. Теплообмен в этих областях не зависит от времени и полностью определяется течением среды, то есть задача становится стационарной с учётом конвективного переноса теплоты по оси $x$.

Подобное разделение решения нестационарной задачи конвективного теплообмена при течении жидкостей в трубах и плоских каналах на стационарную и нестационарную составляющие физически обосновано и в других работах $[3,4]$.

В данном случае наибольшую трудность представляет нахождение решения стационарной задачи (задачи Гретца-Нуссельта). Её математическая постановка в данном случае имеет вид

$$
\begin{gathered}
\left(1-y^{2}\right) \frac{\partial \Theta(y, x)}{\partial}=\frac{\partial^{2} \Theta(y, x)}{\partial y^{2}}+\frac{1}{y} \frac{\partial \Theta(y, x)}{\partial y}, \quad x>0,0 \leqslant y<1 \\
\Theta(y, 0)=0 ; \quad \frac{\partial \Theta(0, x)}{\partial y}=0 ; \quad \Theta(1, x)=1 .
\end{gathered}
$$

Следуя методу Фурье, решение задачи (13), (14) будем искать в виде

$$
\Theta(y, x)=\varphi(x) \psi(y)
$$

Подставляя (15) в (13), находим

$$
\begin{gathered}
\frac{d \varphi(x)}{d x}+\mu \varphi(x)=0 \\
\frac{d^{2} \psi(y)}{d y^{2}}+\frac{1}{y} \frac{d \psi(y)}{d y}+\mu\left(1-y^{2}\right) \psi(y)=0
\end{gathered}
$$

где $\mu$ - некоторая постоянная.

Решение уравнения (16) известно и имеет вид

$$
\varphi(x)=A \exp (-\mu x)
$$

где $A-$ неизвестный коэффициент.

Удовлетворяя (15) двум последним условиям из (14), получаем

$$
\frac{\partial \psi(0)}{\partial y}=0, \quad \psi(1)=0 .
$$

Уравнение Бесселя (17) и граничные условия (19) представляют собой задачу Штурма-Лиувилля. Её точное аналитическое решение, полученное классическими методами, содержит функции Бесселя различного порядка (нулевого и $1 / 3$ ), и к тому же решение выражается различными формулами для различных участков жидкости по радиальной координате $y$. В частности, рассматриваются три участка: вблизи оси трубы (для малых $y$ ), вблизи стенки (для больших $y$ ), для средних y [3]. Использование таких решений для инженерных приложений затруднительно, так как область определения 
каждого из этих трёх решений по радиальной координате не задана, поэтому возникают трудности определения координат точек их сопряжения.

Найдём приближённое аналитическое решение задачи (17), (19). Искомое решение принимается в виде

$$
\psi(y)=B_{0}+\sum_{i=1}^{n} B_{i} y^{i+1}
$$

где $B_{i}$ - неизвестные коэффициенты, определяемые из основных граничных условий (19) и некоторых дополнительных условий. Далее будем полагать, что $n=48$.

Первое дополнительное условие следует из первого условия (19):

$$
\psi(0)=\text { const }:=1 \text {. }
$$

Подставляя (20) в (21), находим $B_{0}=1$.

В качестве других дополнительных условий будем использовать соотношения, получаемые путём удовлетворения решением (20) уравнения (17) в отдельных произвольно выбранных точках переменной $y$ (они называются дополнительными условиями, так как выполняются не на границах, а в промежутке $0<y<1$ ). Для получения дополнительных условий потребуем, чтобы соотношение (20) удовлетворяло уравнению (17) в следующих точках переменной $y: y=1 / 48,2 / 48, \ldots, 47 / 48$. Ещё одно уравнение получим подстановкой (20) во второе граничное условие (19) (отметим, что первое граничное условие (19) соотношением (20) удовлетворяется), и, таким образом, всего будем иметь 49 алгебраических линейных уравнений относительно такого же количества неизвестных коэффициентов $B_{i}$.

После определения коэффициентов $B_{i}$ из решения системы, включающей 49 алгебраических линейных уравнений, и подстановки их в (20) это соотношение будет удовлетворять основным граничным условиям (19) и уравнению (17) в отмеченных выше 47 точках переменной $y$. Отметим, что в соотношении (20) неопределёнными оказываются собственные числа $\mu_{k}$. Для их нахождения потребуем, чтобы соотношение (20) удовлетворяло уравнению (17) во всей области изменения переменной $y(0 \leqslant y \leqslant 1)$. Для этого найдём интеграл взвешенной невязки уравнения (17):

$$
\begin{aligned}
\int_{0}^{1}\left[\frac{d^{2}}{d y^{2}}\left(1+\sum_{i=1}^{n} B_{i} y^{i+1}\right)+\frac{1}{y} \frac{d}{d y}(1\right. & \left.+\sum_{i=1}^{n} B_{i} y^{i+1}\right)+ \\
& \left.+\mu\left(-y^{2}\right)\left(1+\sum_{i=1}^{n} B_{i} y^{i+1}\right)\right] d y=0 .
\end{aligned}
$$

После вычисления интегралов в (22) относительно собственных чисел $\mu_{k}$ получаем алгебраическое уравнение десятой степени, из решения которого находим $\mu_{1}=7,3135869 ; \mu_{2}=44,609461 ; \mu_{3}=113,92103 ; \mu_{4}=215,24054$; $\mu_{5}=348,564115 ; \mu_{6}=513,8899 ; \mu_{7}=711,215 ; \mu_{8}=940,70 ; \mu_{9}=1202,9 ;$ $\mu_{10}=1465,5$. Точные значения собственных чисел [3]: $\mu_{1}=7,3135868 ; \mu_{2}=$ $=44,609460 ; \mu_{3}=113,92104 ; \mu_{4}=215,24054 ; \mu_{5}=348,56412 ; \mu_{6}=513,89$; $\mu_{7}=711,217 ; \mu_{8}=940,54 ; \mu_{9}=1201,8 ; \mu_{10}=1495,2$. 
Высокая точность определения собственных чисел объясняется тем, что соотношение (20) удовлетворяет дифференциальному уравнению (17) в 47 точках переменной $y$.

Собственные функции по найденным значениям собственных чисел находятся из (20). Подставляя (18), (20) в (7) находим

$$
\Theta(y, x)=A \exp (-\mu x)\left(B_{0}+\sum_{i=1}^{n} B_{i} y^{i+1}\right) .
$$

Каждое частное решение (23) удовлетворяет двум последним граничным условиям (14) и уравнению (13) во всех 47 точках пространственной переменной $y$, в которых было выполнено уравнение (17). Однако ни одно из этих частных решений не удовлетворяет первому условию (14). Для его выполнения составим сумму частных решений

$$
\Theta(y, x)=\sum_{k=1}^{m} A_{k} \exp \left(-\mu_{k} x\right)\left(B_{0}+\sum_{i=1}^{n} B_{i} y^{i+1}\right)
$$

где $m=10$ - количество собственных чисел (десятое приближение).

Неизвестные коэффициенты $A_{k}$ найдём так, чтобы соотношение $(24)$ удовлетворяло первому условию (14) в десяти точках (по числу собственных чисел) пространственной переменной $y: y_{1}=0, y_{2}=1 / 10, y_{3}=2 / 10, \ldots$, $y_{10}=9 / 10$. Подставляя (24) в первое условие (14) и записывая полученное соотношение в десяти точках переменной $y$, относительно неизвестных коэффициентов $A_{k}$ получаем систему алгебраических линейных уравнений

$$
\sum_{k=1}^{10} A_{k} \exp \left(-\mu_{k} x\right)\left(B_{0}+\sum_{i=1}^{48} B_{i} y_{j}^{i+1}\right)=0, \quad j=1,2, \ldots, 10
$$

Из решения системы уравнений (25) находим $A_{1}=1,47205 ; A_{2}=-0,82047$; $A_{3}=0,56376 ; A_{4}=-0,53858 ; A_{5}=0,28605 ; A_{6}=-0,47515 ; A_{7}=0,79096 ;$ $A_{8}=0,91147 ; A_{9}=0,49147 ; A_{10}=-1,68158$.

После определения неизвестных коэффициентов $A_{k}$ решение задачи (13), (14) в замкнутом виде находится из (24). Это решение в 47 точках пространственной переменной у удовлетворяет уравнению (13), в десяти точках переменной $y$ - первому граничному условию из (14) и также удовлетворяет двум последним граничным условиям (14).

На рис. 2 сплошными линиями приведены результаты расчётов по формуле (24) при $m=10$, штриховыми линиями - точное решение [3], пунктирными линиями - результаты расчётов по методу конечных разностей с использованием метода прогонки. Анализ представленных результатов позволяет сделать вывод, что полученные по формуле (24) значения температур для $x \geqslant 0,01$ практически совпадают с точными, а при $x=0,005$ расхождение между полученным и точным решением не превышает $2 \%$.

Выводы. Получено приближённое аналитическое решение задачи теплообмена при течении жидкости в круглой трубе, позволяющее для инженерных приложений с достаточной точностью определять температурное состояние 


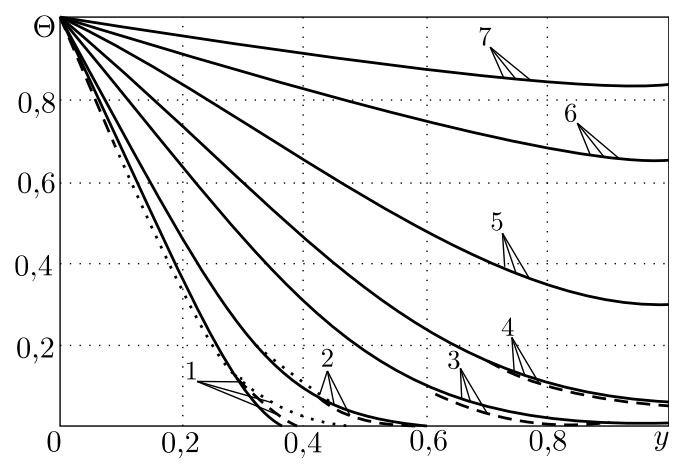

Рис. 2. Распределение температуры в цилиндрическом канале: сплошная линия - расчёт по формуле (24); штриховая линия - точное решение; пунктирная линия - метод конечных разностей; 1) $x=0,005,2) x=0,01,3) x=0,025,4) x=0,05,5) x=0,1,6) x=0,2,7) x=0,3$

жидкости от продольной пространственной переменной $x \geqslant 0,005$. В отличие от точного решения, полученное решение не содержит специальных функций и имеет вид алгебраических полиномов с коэффициентами, экспоненциально стабилизирующимися во времени.

Получение решения для достаточно малых значений продольной переменной $x$ оказалось возможным благодаря использованию дополнительных условий. Для получения этих условий используется искомое в виде полиномов решение дифференциального уравнения (17) задачи Штурма-Лиувилля, которое должно удовлетворять уравнению в заданном числе точек поперечной пространственной переменной. Использование этих условий также приводит к удовлетворению искомым решением исходного дифференциального уравнения (13) краевой задачи Гретца-Нуссельта в указанных точках.

\section{БИБЛИОГРАФИЧЕСКИЙ СПИСОК}

1. L. Graetz, "Ueber die Wärmeleitungsfähigkeit von Flüssigkeiten. 2. Abhandlung" // Annalen der Physik und Chemie, 1885. Vol.25, no. 7. Pp. 337-357.

2. W. Nusselt, "Die Abhängigkeit der Wärmeübergangszahl von der Rohrlänge" // Z. Ver. Deut. Ing., 1910. Vol. 54. Pp. 1154-1158.

3. Б. С. Петухов, Теплообмен и сопротивление при ламинарном течении жидкости в трубах. М.: Энергия, 1967. 411 с. [B. S. Petukhov, Heat transfer and resistance at laminar flow of fluids in pipes. Moscow: Energiya, 1967. 411 pp.]

4. П. В. Цой, Системные методы расчета краевых задач тепломассопереноса. М.: МЭИ, 2005. 568 c. [P. V. Tsoi, System Methods of Calculation of Boundary-Value Problems of Heat- and Mass Transfer. Moscow: MÉI, 2005. 566 pp.]

5. В. А. Кудинов, Э. М. Карташов, Е. В. Стефанюк, Техническая термодинамика и теплопередача. М.: Юрайт, 2012. 559 с. [V. A. Kudinov, É. M. Kartashov, E. V. Stefanyuk, Engineering Thermodynamics and Heat Transfer. Moscow: Yurayt, 2012. 559 pp.]

6. В. А. Кудинов, Е. В. Стефанюк, М. С. Антимонов, "Аналитические решения задач теплообмена при течении жидкости в плоскопараллельных каналах на основе определения фронта температурного возмущения" // Инженерно-физический журнал, 2007. T. 80, № 5. C. 176-186; англ. пер.: V. A. Kudinov, E. V. Stefanyuk, M. S. Antimonov, "Analytical solutions of the problems of heat transfer during liquid flow in plane-parallel channels by determining the temperature perturbation front" // J. Eng. Phys. Thermophys., 2007. Vol. 80, no. 5. Pp. 1038-1049.

7. Стефанюк E. В., Кудинов В. А., "Дополнительные граничные условия в нестационарных задачах теплопроводности" // Теплофизика высоких температур, 2009. Т. 47, № 2. 
C. 269-282; англ. пер.: E. V. Stefanyuk, V. A. Kudinov, "Additional boundary conditions in nonstationary problems of heat conduction" // High Temp., 2009. Vol. 47, no. 2. Pp. 250262.

Поступила в редакцию $21 / \mathrm{II} / 2013$;

в окончательном варианте - 24/VII/2013.

MSC: 80A20

\section{NON-STATIONARY HEAT EXCHANGE IN CYLINDRICAL CHANNEL AT LAMINAR FLOW OF FLUIDS}

\section{A. V. Eremin, E. V. Stefanyuk, A. Yu. Rassypnov, A. E. Kuznetsova}

Samara State Technical University,

244, Molodogvardeyskaya st., Samara, 443100, Russia.

E-mails: a.v.eremin@list.ru, stef-kate@yandex.ru, kuznetsovaae@rambler.ru

Using double integral Laplace-Carson transformation and orthogonal method of Bubnov-Galyorkin, the analytical solution of the non-stationary problem of heat transfer in a cylindrical channel in the laminar flow of fluids was obtained. It has two components: stationary and non-stationary, each part has application only in a certain range of temporal and spatial coordinates. For the stationary Graetz-Nusselt problem on the basis of introduction of the temperature perturbation front and additional boundary conditions it was managed to find an analytical solution that allows the assessment of liquid thermal state with small values of spatial variable, directed along the stream flow. It is not possible to obtain such results using the well-known exact analytical methods because of the poor convergence of infinite series of received solutions.

Keywords: cylindrical channel, Graetz-Nusselt problem, integral Laplace-Carson transformation, Fourier method, additional conditions, Sturm-Liouville problem, analytical solution.

Original article submitted 21/II/2013; revision submitted $24 / \mathrm{VII} / 2013$.

Anton V. Eremin, Postgraduate Student, Dept. of Theoretical Basis of Heat Engineering \& Flow Mechanics. Ekaterina V. Stefanyuk (Dr. Techn. Sci.), Associate Professor, Dept. of Theoretical Basis of Heat Engineering \& Flow Mechanics. Alexander Yu. Rassypnov, Postgraduate Student, Dept. of Theoretical Basis of Heat Engineering \& Flow Mechanics. Anastasiya E. Kuznetsova, Postgraduate Student, Dept. of Theoretical Basis of Heat Engineering \& Flow Mechanics. 